\title{
Estimation of fissile material content in irradiated In-Pile Sections using neutron coincidence counters
}

\author{
Riccardo Rossa*, Alessandro Borella, Sven Boden, Wouter Broeckx \\ SCK-CEN \\ *rrossa@sckcen.be
}

\begin{abstract}
A set of In-Pile Sections (IPS) has been irradiated in the BR2 reactor at $\mathrm{SCK} \cdot \mathrm{CEN}$ in Belgium during the 1970's and 1980's. The primary goal of the IPS was to replicate the thermohydraulic loop of a sodium-cooled fast reactor in order to study severe accident scenarios. The top part of the IPS contained the sodium-cooled loop whereas the lower part contained the fuel element. Due to the experimental conditions, the rupture of the fuel pins contained in the IPS occurred and fuel fragments may have been deposited in the rest of the IPS loop. The part of the IPS containing the fuel pins has been cut from the rest of the IPS and underwent post-irradiation examinations at specialized EU laboratories, while the top parts remained stored at SCK•CEN. To prepare for future transport, dismantling and conditioning, a reliable estimation of the total fissile content in the stored parts of the IPS is indispensable.

In this framework, two IPS were measured with a Canberra WM3400 neutron coincidence counter with customized electronics. The measurements of the IPS were challenging due their length (roughly $6 \mathrm{~m}$ ) and intense gamma-ray radiation background. For each IPS an axial scan was carried out with a series of short measurements $(600-700 \mathrm{~s}$ each) recording the Totals rate and Reals rate. Based on the results of the axial scans, measurements with longer measurement time were conducted for the axial positions with the larger values of Reals rates.

A system of equations was then established to quantify the ${ }^{240} \mathrm{Pu}$ content in the different sections of the IPS from the Reals rates in each measurement position and account for cross-talk between the neutron emission associated to the different sections. A set of Monte Carlo simulations was carried out to estimate the probability to record a Real count in the detector due to spontaneous fission events occurring in a given section of the IPS. The ${ }^{240} \mathrm{Pu}$ content in each section of the IPS was calculated by combining the measured Reals rates and the detection probabilities calculated with the simulations. The total fissile content in the IPS was then determined with scaling factors based on burnup calculations for the irradiated fuel assemblies in the IPS. The results indicate that both IPS measured with the neutron coincidence counters have a fissile content lower than the limit for transport. It is expected that the envisaged segmentation of the IPS in shorter sections required to fit into $200 \mathrm{~L}$ drums will provide an additional safety margin on this limit.
\end{abstract}

Keywords - Neutron coincidence counters, decommissioning, In-Pile Sections, fissile material, non-destructive assay

R. Rossa (rrossa@sckcen.be), A. Borella (aborella@sckcen.be), S. Boden (sboden@sckcen.be), and W. Broeckx (wbroeckx@sckcen.be) are with the

\section{INTRODUCTION}

T $\mathrm{N}$ the frame of research on the safety analysis of Liquid Metal 1 Fast Breeder Reactors, several research projects have been performed at the SCK $\bullet \mathrm{CEN}$. A set of so-called In-Pile Sections (IPS) has been irradiated in the BR2 reactor during the 1970's and 1980's, testing the behavior of fuel pins during severe accidents [1]. Due to the experimental conditions, the rupture of the fuel pins contained in the IPS occurred and fuel fragments may have been deposited in the rest of the IPS loop. The part of the IPS containing the fuel pins has been cut from the rest of the IPS and underwent post-irradiation examinations at specialized EU laboratories, while the top parts remained stored at $\mathrm{SCK} \cdot \mathrm{CEN}$.

To prepare for future transport, dismantling and conditioning, a reliable estimation of the total fissile content in the stored parts of the IPS is indispensable. Two IPS were measured with a Canberra WM3400 [2] neutron coincidence counter with customized electronics [3].

Irradiated material such as the IPS emits neutrons by $(\alpha, n)$ reactions, spontaneous fission, or induced fission. In the first case only one neutron is emitted per reaction, whereas usually more than one neutron is emitted in the fission process. Coincidence counters exploit this difference to estimate the presence of fissile material in the measured sample [4].

In this paper the Canberra WM3400 detector is described in Section II with the associated Monte Carlo model, whereas the geometry of the IPS is described in Section III. The details of the measurement campaign and the approach used for the estimation of the total fissile content in the IPS are discussed in Section IV. The results of the measurements are presented in Section V and the conclusion is drawn in Section VI.

\section{CANBERRA WM3400 DETECTOR}

\section{A. Detector design}

Two used Canberra WM3400 coincidence counters were purchased for the measurement of the IPS. A picture of the WM3400 detector with a ${ }^{252} \mathrm{Cf}$ source used for testing is shown in Figure 1.

The dimensions of the detector are $104 \mathrm{~cm}$ (height) $\times 41 \mathrm{~cm}$ (width) $\times 16 \mathrm{~cm}$ (depth), and the total weight is approximately 
$50 \mathrm{~kg}$. An aluminum cover protects the internal parts of the detector.

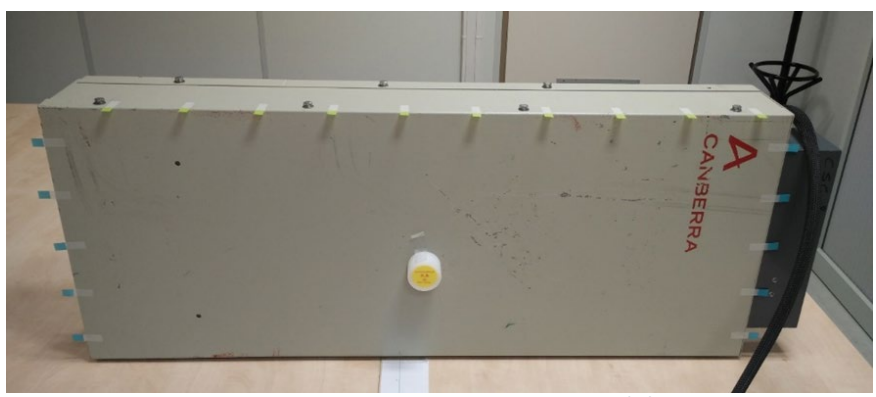

Figure 1: Picture of the WM3400 detector with a ${ }^{252} \mathrm{Cf}$ source used for testing.

The WM3400 detector contains six ${ }^{3} \mathrm{He}$ tubes with active length of $91.44 \mathrm{~cm}$ and diameter of $2.54 \mathrm{~cm}$. The ${ }^{3} \mathrm{He}$ tubes are longer than the active length, and an air gap of $7.4 \mathrm{~cm}$ and $1.27 \mathrm{~cm}$ precedes and follows the section filled by gas, respectively. A Cd layer of $1 \mathrm{~mm}$ covers five sides of the detector according to the arrangement observed by opening the outer aluminum layer of the WM3400 detector. Only the side of the detector facing the radioactive source is not covered by $\mathrm{Cd}$.

The original electronics of the detector was replaced to reduce the sensitivity to gamma-rays and time fluctuations observed during a previous measurement campaign. The new electronic system is described in detail in [3].

The WM3400 detector was connected to a MCA-527 multichannel analyzer with upgraded firmware to record the time stamps of the neutron detections [5]. The time stamps are processed off-line to determine Totals $(T)$, Reals+Accidentals $(R+A)$, and Accidentals $(A)$ counts and the multiplicity distribution [4] as well as to remove system instabilities due to high voltage fluctuations or interferences.

\section{B. Monte Carlo model}

A Monte Carlo model with the MCNPX code [6] was developed from the technical characteristics of the WM3400 coincidence counter. Figure 2 shows the inner section of the model to highlight the position of the ${ }^{3} \mathrm{He}$ tubes and the $\mathrm{Cd}$ layers within the polyethylene.

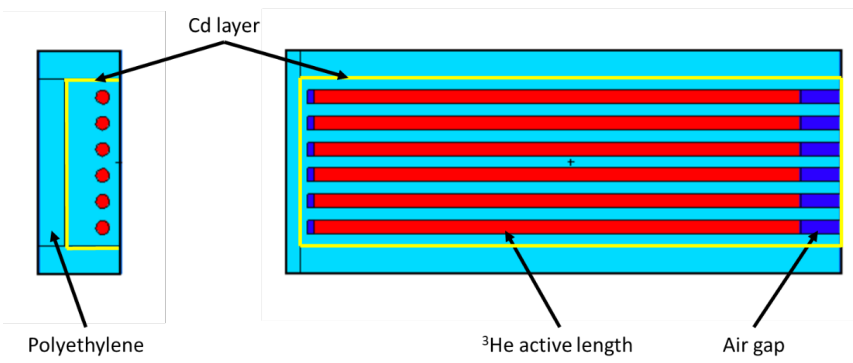

Figure 2: 2D-views of the Canberra WM3400 counter developed with the MCNPX Monte Carlo code.

The MCNPX-PoliMi V.2.0 code [7] and the MPPost postprocessor [8] were used for the data analysis to obtain the RossiAlpha and multiplicity distributions. The MCNPX-PoliMi code requires to perform the Monte Carlo simulation in the so-called analog mode, in which each particle has a weight equal to 1 . This is set by the following lines:

$$
\begin{array}{llllll}
\text { CUT:N } & 2 j & 0 & 0 \\
\text { PHYS:N } & j & 100 & 3 j & -1
\end{array}
$$

The source term in the MCNPX-PoliMi code was defined with the IPOL card.

$$
\text { IPOL + } 22002 j 6201202203204205206
$$

The first term $(+2)$ in the IPOL card sets ${ }^{240} \mathrm{Pu}$ spontaneous fission as the modelled source term, while the second term (2) defines the angular distribution in case of induced fission. The third term $(0)$ is used to neglect the correlation between neutron and photon production, and the fourth term $(0)$ applies no delay in the photon emission following a fission event. Two other entries are set to default values $(2 j)$, and finally the six cells with the ${ }^{3} \mathrm{He}$ tubes are listed $(201, \ldots, 206)$.

In addition, the threshold for neutron energy release to be collected was set to $1 \mathrm{keV}$ using the first term of the RPOL card. The second term of the $R P O L$ card is the threshold for gammarays and was kept to the default value $(j)$.

$$
\text { RPOL } 0.001 j
$$

The neutron flux tally (so-called F4 type) was calculated for each ${ }^{3} \mathrm{He}$ tube with the FM treatment [6] to simulate the detector response taking into account the $(n, p)$ reaction cross-section for ${ }^{3} \mathrm{He}$ and a ${ }^{3} \mathrm{He}$ pressure of 4 atm [2].

The $(R+A)$ and $(A)$ scalers from the Rossi-Alpha distribution were calculated with the MPPost post-processor from the output file of the Monte Carlo simulation specifying a pre-delay time of $4.5 \mu \mathrm{s}$, a gate width of $64 \mu \mathrm{s}$, and a long delay of 1000 $\mu \mathrm{s}$.

\section{IN-PILE SECTIONS}

Two IPS (named MFBS6 and MOL7C7) were measured in May-June 2018 with one WM3400 neutron coincidence counter. The MFBS series of IPS were irradiated in the BR2 reactor during the 1970's, whereas the MOL7C IPS were used in the period 1977-1989 [1]. Some information about the IPS designs are included in Table 1.

Table 1: Design information of the IPS taken from [1]

\begin{tabular}{|l|l|l|}
\hline $\begin{array}{l}|c| \\
\text { Irradiation } \\
\text { year }\end{array}$ & 1971 & \multicolumn{1}{|c|}{ MOL7C7 } \\
\hline Fuel type & $\begin{array}{l}\text { 6 double MOX pins; } \\
1 \text { double } \text { UO }_{2} \text { pin }\end{array}$ & 30 MOX pins \\
\hline Length & $500 \mathrm{~cm}$ (approx.) & $608 \mathrm{~cm}$ \\
\hline Diameter & $36 \mathrm{~cm}$ (maximum) & $\begin{array}{l}74 \mathrm{~cm} \text { (body) } \\
165 \mathrm{~cm} \text { (maximum) }\end{array}$ \\
\hline
\end{tabular}

During the measurement campaign we noticed that the actual length of the MFBS6 IPS appeared to be longer than the $500 \mathrm{~cm}$ reported in literature. Both IPS therefore were modelled in MCNPX as cylinders with total length of $600 \mathrm{~cm}$. The diameter of each IPS was varied according to the literature data. 
A thickness of $5 \mathrm{~mm}$ of stainless steel was chosen as outer case of the IPS. The inner section of the IPS was a cylinder filled with a homogeneous material with same composition of stainless steel, but density reduced to $1.955 \mathrm{~g} / \mathrm{cm}^{3}(25 \%$ of normal density). The reduction in density took into account that the IPS is not completely filled with material. Both IPS presented a lead shielding on the top part and this was included in the MCNPX models as well. The thickness of the lead shielding was $2 \mathrm{~cm}$ for the MFBS6 IPS and $10 \mathrm{~cm}$ for the MOL7C7 IPS.

\section{MEASUREMENT CAMPAign}

\section{A. Measurement setup}

Both IPS are stored under water in the BR2 channel at SCK $•$ CEN so the WM3400 detector was placed horizontally on the side of the channel. A picture of the measurement setup is shown in Figure 3. The position of the detector was fixed for the whole measurement campaign and for each measurement the IPS was moved to the desired height and distance from the detector.

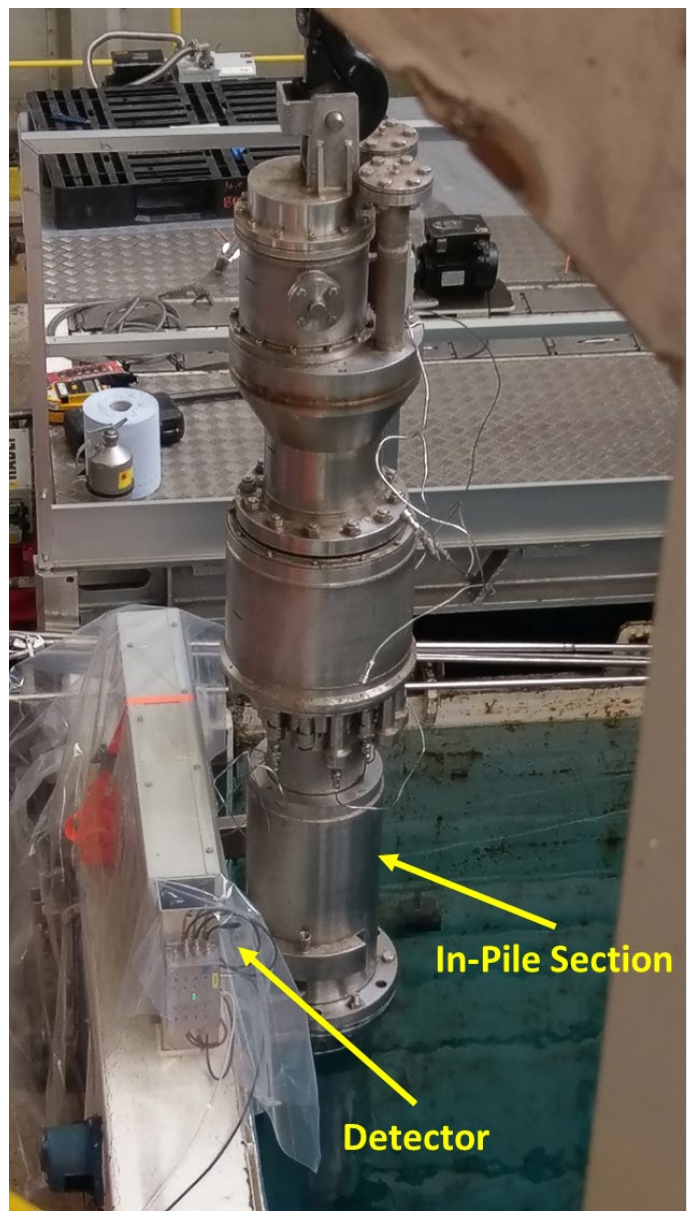

Figure 3: Picture of the measurement setup.

A set of short measurements (600-700 s each) was carried out to obtain an axial profile of the IPS both in terms of Totals rate and Reals rate.

The starting position (POSO1) was approximately set with the IPS completely out of the water and the bottom of the IPS at the same height of the neutron detector. The IPS was then lowered in steps of $40 \mathrm{~cm}$ until it was entirely immersed in water. A set of longer measurements was then carried out for the positions with the largest Real rates recorded during the axial scan.

\section{B. Estimation of the fissile material content}

A system of equations was established to first quantify the ${ }^{240} \mathrm{Pu}$ content in the different sections of the IPS from the Reals counts in each position.

$$
\left\{\begin{array}{c}
R_{1}=S_{1} P_{1,1}+S_{2} P_{1,2}+S_{3} P_{1,3}+\cdots+S_{15} P_{1,15} \\
\cdots \\
\cdots \\
R_{15}=S_{1} P_{15,1}+S_{2} P_{15,2}+S_{3} P_{15,3}+\cdots S_{15} P_{15,15}
\end{array}\right.
$$

For each measurement position $i$, the Reals count $R_{i}$ is related to the ${ }^{240} \mathrm{Pu}$ mass in each section $S_{i}$, through the importance function $P_{i, j}$ which is the probability to have a Real count in section $j$ from a ${ }^{240} \mathrm{Pu}$ spontaneous fission event in section $i$. The importance function was calculated with a set of Monte Carlo simulations with the WM3400 detector facing the IPS in section $i$ and the neutron source term only in section $j$. Different simulations were carried out to take into account the distance between the detector and the IPS.

Only the three sections above and below the detector position were considered in the analysis, since it was assumed that the importance function was negligible for the positions further away from the detector. This assumption is supported by the consideration that the section facing the detector contributes for about $50 \%$ of the Totals rate and $75 \%$ of the Reals rate considering all source positions. Considering also the positions above and below the detectors the combined values account for about $88 \%$ and $98 \%$ of the Totals rate and Reals rate, respectively.

The Reals rates are then converted in ${ }^{240} \mathrm{Pu}$ mass using the ${ }^{240} \mathrm{Pu}$ specific neutron emission [9]. This conservative approach assumes that the measured Reals rates are only due to ${ }^{240} \mathrm{Pu}$. The total fissile material content in the IPS was finally determined with scaling factors based on burnup calculations for the irradiated fuel pins in the IPS [1].

\section{RESULTS}

\section{A. MFBS6}

The Totals rate and Reals rate measured during the axial scan and long measurements are shown in Figures 4 and 5, respectively. The plots include also the uncertainty due to counting statistics.

The trends for the Totals rate and Reals rate are similar, with the maximum rates that were obtained in central sections of the IPS (POS04, POS07, and POS08). For these positions the values for the long measurement were larger than the values obtained in the quick axial scan. The values of the Reals rate in POS13 are also larger than in the neighboring positions, but this is probably due to the presence of lead shielding in that axial region of the IPS. Cosmic radiation induces spallation reactions in lead which cannot be easily distinguished from spontaneous fission events. 


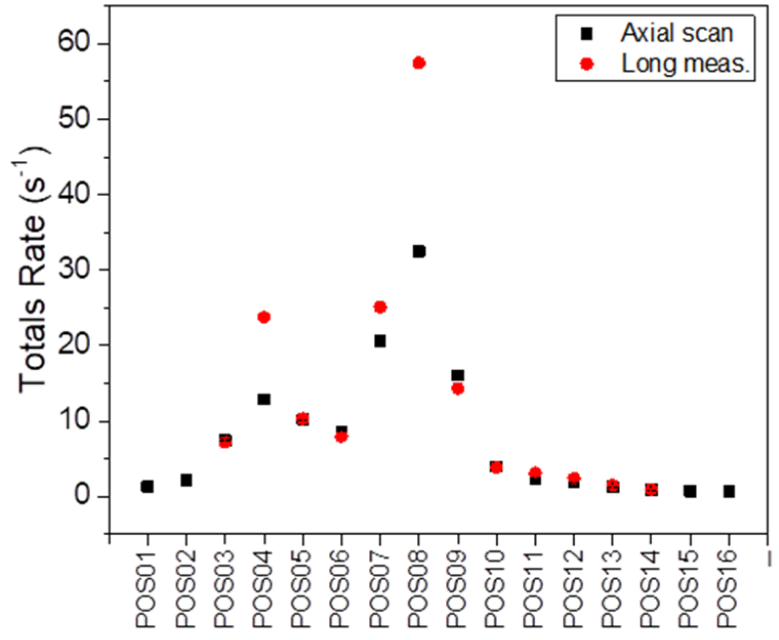

Figure 4: Measured Totals rate from the axial scan and the long measurements of the MFBS6 IPS.

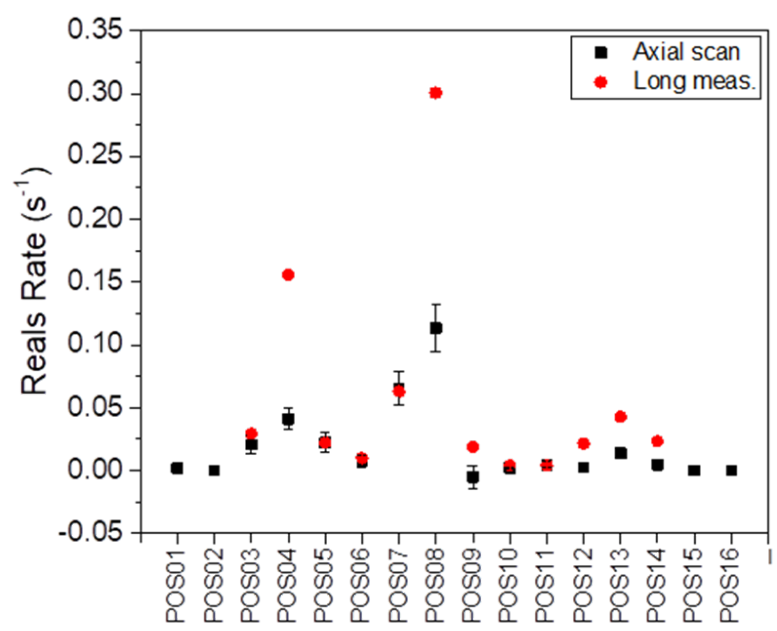

Figure 5:Measured Reals rate from the axial scan and the long measurements of the MFBS6 IPS.

The results from the long measurements are reported in Table 2 in terms of Totals, Reals, and Accidentals rates. The estimate of the ${ }^{240} \mathrm{Pu}$ content in each section is also included. According to these measurements the ${ }^{240} \mathrm{Pu}$ content is concentrated in the central section of the IPS, with a total ${ }^{240} \mathrm{Pu}$ mass for the whole IPS of $0.8 \mathrm{~g}$. Using the scaling factors based on the burnup calculations the estimated total fissile material content in the IPS is $14 \mathrm{~g}$. This estimate is comparable to a conservative upper limit based on the ${ }^{137} \mathrm{Cs}$ content [1].

The total fissile material estimated in the IPS is lower than the limit of $45 \mathrm{~g}$ set by current regulation for transport [10], and the further segmentation of the IPS in several 220L drums will introduce an additional margin.

\section{B. $M O L 7 C 7$}

The Totals rate and Reals rate measured during the axial scan and long measurements are shown in Figures 6 and 7, respectively. The plots include also the uncertainty due to counting statistics.

Also for this IPS the Totals rate and Reals rate are strongly correlated, but in this case the maximum Totals and Reals rates were obtained at the extremities of the IPS (POSO2, POS11, and $P O S 12)$. The values for the long measurements are comparable to the values obtained in the quick axial scan, with the Totals rates that are outside the statistical uncertainty only for a couple of positions. One should stress that the positioning of the IPS was not necessarily the same for the axial scan and the long measurements. As for the previous IPS, the increase of the Reals rate in POS11 and POS12 are probably due to the presence of lead shielding in that axial region. The Reals rates obtained for the MOL7C7 IPS are in general lower than those obtained during the MFBS6 measurements.

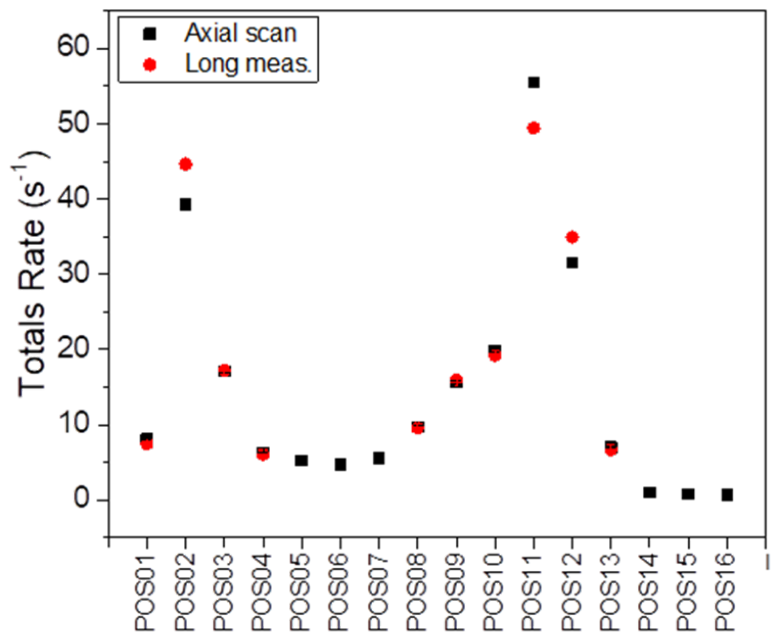

Figure 6: Measured Totals rate from the axial scan and the long measurements of the MOL7C7 IPS.

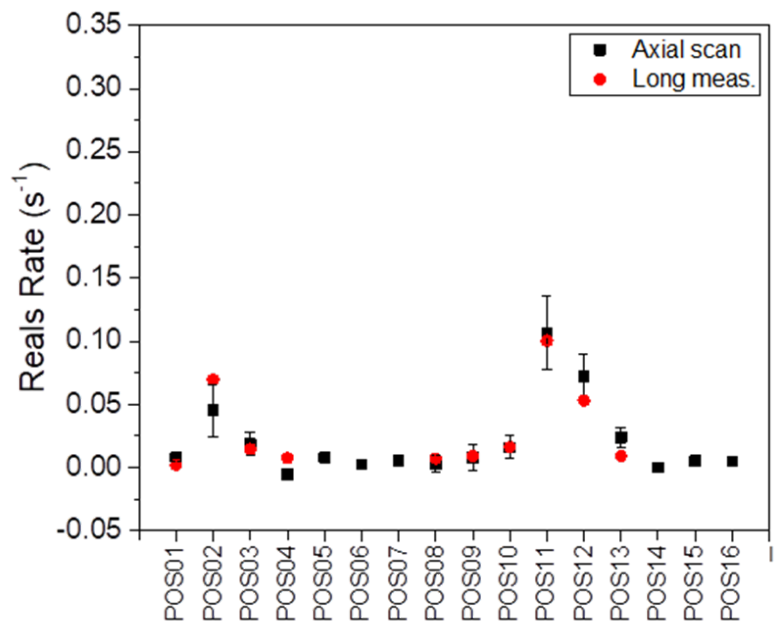

Figure 7:Measured Reals rate from the axial scan and the long measurements of the MOL7C7 IPS.

The results from the long measurements are reported in Table 3 in terms of Totals, Reals, and Accidentals rates. The estimate of the ${ }^{240} \mathrm{Pu}$ content in each section is also included. According to these measurements the ${ }^{240} \mathrm{Pu}$ content is concentrated at the extremities of the IPS, with a total ${ }^{240} \mathrm{Pu}$ mass for the whole IPS of $1.8 \mathrm{~g}$. Using the scaling factors based on the burnup calculations the estimated total fissile material content in the IPS is $22 \mathrm{~g}$. This estimate is comparable to the detection limit 
based on an independent total neutron counting using a Berthold LB6411 portable neutron dose rate probe that is used for ambient dose equivalent for neutrons [1].

\section{CONCLUSION}

A Canberra WM3400 neutron coincidence counter was used for the measurement of two irradiated IPS in view of their future transport, dismantling and conditioning.

In addition, a Monte Carlo model of the detector and of the IPS was developed and used for the estimation of the total fissile material in the IPS.

The IPS were measured with a short axial scanning and long measurements for the positions with the largest values of the Reals rate. The results obtained during the two sets of measurements were comparable for both IPS.

The total fissile material estimated for the two IPS is $14 \mathrm{~g}$ for the MFBS6 and $22 \mathrm{~g}$ for the MOL7C7. Both estimates are in line with results obtained with other measurement techniques. For both IPS the total fissile material estimated is lower than the limit of $45 \mathrm{~g}$ set by current regulation for transport, and the further segmentation of the IPS in several 220L drums will introduce an additional margin.

\section{REFERENCES}

[1] S. Boden, et al., "Remaining In-Pile Sections of the Fast Breeder Reactor Programme - Estimation of the fissile material present" SCK •CEN internal report I-0642, 2018
[2] http://www.canberra.com/products/waste safeguard systems/pdf/ WM3400-SS-0258.pdf. Last accessed 05/03/2018.

[3] A. Borella et al, "Improved electronics for $3 \mathrm{He}$ based neutron counters" to be presented at the 2019 ANIMMA conference, 2019.

[4] N. Ensslin, "Principles of Neutron Coincidence Counting" in Passive Nondestructive Assay of Nuclear Materials, NuREG/CR-5550, LA-UR-90732, 1991

[5] https://www.gbs-elektronik.de/media/download_gallery/mca527_neutron .pdf. Last accessed 19/04/2018

[6] D. Pelowitz, "MCNPX user's manual version 2.7.0". Los Alamos National Laboratory LACP-11-00438, 2011.

[7] E. Padovani, et al., "MCNPX-PoliMi User's Manual”. Radiation Safety Information Computational Center, Oak Ridge National Laboratory, 2012.

[8] E. Miller, et al., "MCNPX-PoliMi Post-Processor (MPPost) Manual". Radiation Safety Information Computational Center, Oak Ridge National Laboratory, 2012.

[9] A. J. Koning, et al., "Status of the JEFF Nuclear Data Library". Proceedings of the International Conference on Nuclear Data for Science and Technology, 2010, pp.1057-1062.

[10] "European Agreement concerning the International Carriage of Dangerous Goods by Road". United Nations Economic Commission for Europe (UNECE), 2007.

Table 2: Results from the long measurements and estimate of the ${ }^{240} \mathrm{Pu}$ content for each measurement position of the MFBS6 IPS.

\begin{tabular}{|c|r|r|r|r|r|r|}
\hline Position ID & $\begin{array}{c}\text { Meas. time } \\
(\mathrm{s})\end{array}$ & $\begin{array}{c}\gamma \text {-ray dose rate } \\
(\mu \mathrm{Sv} / \mathrm{h})\end{array}$ & $\begin{array}{c}\text { Totals rate } \\
\left(\mathrm{s}^{-1}\right)\end{array}$ & $\begin{array}{c}\text { Reals rate } \\
\left(\mathrm{s}^{-1}\right)\end{array}$ & $\begin{array}{c}\text { Accidentals rate } \\
\left(\mathrm{s}^{-1}\right)\end{array}$ & $\begin{array}{r}240 \mathrm{Pu} \text { content } \\
(\mathrm{g})\end{array}$ \\
\hline POS03 & 11100 & 7000 & $7.211 \pm 0.025$ & $0.029 \pm 0.002$ & $0.004 \pm 0.001$ & $<0.01$ \\
\hline POS04 & 58380 & 8000 & $23.771 \pm 0.020$ & $0.156 \pm 0.002$ & $0.036 \pm 0.001$ & 0.28 \\
\hline POS05 & 13500 & 21000 & $10.343 \pm 0.028$ & $0.022 \pm 0.002$ & $0.007 \pm 0.001$ & $<0.01$ \\
\hline POS06 & 14220 & 1000 & $7.976 \pm 0.024$ & $0.010 \pm 0.001$ & $0.005 \pm 0.001$ & 0.01 \\
\hline POS07 & 59340 & 15000 & $25.117 \pm 0.021$ & $0.063 \pm 0.002$ & $0.042 \pm 0.001$ & 0.03 \\
\hline POS08 & 211260 & 370 & $58.491 \pm 0.017$ & $0.307 \pm 0.002$ & $0.218 \pm 0.001$ & 0.38 \\
\hline POS09 & 10620 & 8800 & $14.344 \pm 0.037$ & $0.019 \pm 0.002$ & $0.013 \pm 0.001$ & $<0.01$ \\
\hline POS10 & 12347 & 320 & $3.861 \pm 0.018$ & $0.004 \pm 0.001$ & $0.001 \pm<0.001$ & 0.01 \\
\hline POS11 & 14880 & 170 & $3.081 \pm 0.014$ & $0.004 \pm 0.001$ & $0.001 \pm<0.001$ & $<0.01$ \\
\hline POS12 & 14557 & 8 & $2.418 \pm 0.013$ & $0.021 \pm 0.001$ & $0.000 \pm<0.001$ & 0.01 \\
\hline POS13 & 58560 & 3 & $1.523 \pm 0.005$ & $0.045 \pm 0.001$ & $0.000 \pm<0.001$ & 0.07 \\
\hline POS14 & 10020 & 8 & $0.960 \pm 0.010$ & $0.023 \pm 0.001$ & $0.000 \pm<0.001$ & 0.03 \\
\hline
\end{tabular}

Table 3: Results from the long measurements and estimate of the ${ }^{240} \mathrm{Pu}$ content for each measurement position of the MOL7C7 IPS.

\begin{tabular}{|c|r|r|r|r|r|r|}
\hline Position ID & $\begin{array}{c}\text { Meas. time } \\
(\mathrm{s})\end{array}$ & $\begin{array}{l}\gamma \text {-ray dose rate } \\
(\mu \mathrm{Sv} / \mathrm{h})\end{array}$ & $\begin{array}{c}\text { Totals rate } \\
\left(\mathrm{s}^{-1}\right)\end{array}$ & $\begin{array}{c}\text { Reals rate } \\
\left(\mathrm{s}^{-1}\right)\end{array}$ & $\begin{array}{c}\text { Accidentals rate } \\
\left(\mathrm{s}^{-1}\right)\end{array}$ & $\begin{array}{c}240 \mathrm{Pu} \text { content } \\
(\mathrm{g})\end{array}$ \\
\hline POS01 & 12480 & 8 & $7.471 \pm 0.024$ & $0.002 \pm 0.001$ & $0.004 \pm 0.001$ & $<0.01$ \\
\hline POS02 & 60420 & 145 & $44.623 \pm 0.027$ & $0.070 \pm 0.002$ & $0.128 \pm 0.001$ & 0.12 \\
\hline POS03 & 60060 & 40 & $17.234 \pm 0.017$ & $0.015 \pm 0.001$ & $0.019 \pm 0.001$ & $<0.01$ \\
\hline POS04 & 211260 & 20 & $6.056 \pm 0.005$ & $0.008 \pm<0.001$ & $0.002 \pm<0.001$ & 0.01 \\
\hline POS08 & 25140 & 180 & $9.563 \pm 0.020$ & $0.007 \pm 0.001$ & $0.006 \pm<0.001$ & 0.01 \\
\hline POS09 & 25917 & 170 & $16.005 \pm 0.025$ & $0.009 \pm 0.001$ & $0.017 \pm 0.001$ & 0.02 \\
\hline POS10 & 26460 & 90 & $19.198 \pm 0.027$ & $0.016 \pm 0.002$ & $0.024 \pm 0.001$ & $<0.01$ \\
\hline POS11 & 59040 & 3.5 & $49.446 \pm 0.029$ & $0.100 \pm 0.003$ & $0.158 \pm 0.002$ & 0.56 \\
\hline POS12 & 61020 & 8 & $34.923 \pm 0.024$ & $0.053 \pm 0.002$ & $0.079 \pm 0.001$ & \\
\hline
\end{tabular}

